\title{
La profesión del crítico y la vanguardia en la prensa cotidiana (Madrid: 1950-1963)
}

\author{
Begoña fernández Cabaleiro*
}

\begin{abstract}
RESUMEN
ABSTRACT

El ejercicio de la crítica de arte en el Madrid de la posguerra presenta unas características espaciales debido a la particular situación política surgida como resultado de la Guerra Civil. El nuevo régimen que se deseaba establecer tenía unas premisas ideológicas que llevaron al control de la cultura y de los medios para influir en la opinión pública. En los estrechos márgenes resultantes se movían los principales críticos de arte de la prensa cotidiana de los años cincuenta, vinculados de antemano a los postulados ideológicos vigentes.

Sus características y evolución acorde con las necesidades políticas $y$ culturales del país-es analizada en este breve estudio hasta los primeros años sesenta en que se esboza un nuevo sector de la crítica de formación y rasgos diferentes.

The practice of the art critic in Madrid after the Civil War had special characteristics because of the politic situation then. The new political regime wanted to controlate the culture and the public opinion. The main art critics had narrow ways of work about fifties. Their characteristics and evolution acording to the politic and cultural needs in the country - are studied in this work until first sixties when we can find a new way of art critics.
\end{abstract}

* Tercer Ciclo. Departamento de Historia del Arte. UNED. 
Para definir los rasgos de la profesión del crítico de arte en España durante el siglo xx y más específicamente en relación con el desarrollo de la vanguardia, es preciso analizar, aunque solo sea brevemente, los aspectos que rodearon e influyeron de manera fundamental en ambos hechos.

\section{EL NUEVO ESTADO Y LA PRENSA}

El sistema político que gobernó España a partir de 1939 se basaba en la existencia de un único partido bajo la autoridad del Jefe del Estado. En su ideología se mezclaban elementos de la derecha más tradicional con aportaciones del ideario falangista próximos al fascismo europeo. Se pretende crear un nuevo Estado cuyos pilares serán el nacionalismo exaltado y el catolicismo conservador. Frente a enemigos como el liberalismo o el comunismo ateo era necesario consolidar una mentalidad en la que los conceptos de Patria y Dios estuviesen indisolublemente unidos. De ambos había brotado el concepto de Cruzada como justificación de la Guerra Civil. Ahora debian ser fundamento de una nueva sociedad: identidad de lo nacional y lo católico; unidad frente a los separatismos y los partidos; al fondo, el Siglo de Oro y la Cristiandad como modelos ideales, y el fascismo - salvo en lo irreligioso- como modelo actual. Autoridad, jerarquía, servicio, disciplina '.

En esta labor de "reconstrucción» se hizo necesario recurrir a medios rigurosos primero de depuración y después de estrecho control de todos los campos de acción tanto política como social y económica. Un aspecto importante lo constituyó el control de la cultura, pensamiento y opinión pública, elementos fundamentales a la hora de fijar las pautas de desarrollo y conducta en una sociedad. En este sentido son instrumentos básicos la prensa y los medios de comunicación así como todos los campos relacionados con la educación.

Durante los años de la guerra las autoridades franquistas fueron poniendo las bases del sistema informativo al que se quería llegar después de la paz. En realidad fue la Falange la única formación de los sublevados que se ocupó durante la guerra de las tareas de prensa y propaganda. Cuando se nombró

Camón Aznar entrevistado por el diario Madrid en fecha tan avanzada como el 28-V-1963 hacia afirmaciones significativas en este sentido:

- Para usted, ¿Cuál fue nuestro siglo de oro?

- El que se conoce como Siglo de Oro. Hay que intentar llegar ahora al mismo meollo de las cosas. Sobre las inquietudes actuales de nuestro intelectualismo señaló "una preocupación metafísica que puede superar ese intelectualismo que todavia priva" y señalaba "el religioso" como un problema "en primera línea". 
el primer Gobierno, el 31 de enero de 1938, recaen sobre ella las reponsabilidades en este campo. En este gobierno es figura clave Serrano Suñer, quien no aceptó la Secretaría General del Movimiento pero controló los servicios de prensa, radio y propaganda. A él correspondió el patrocinio de la política cultural y la Ley de Prensa del 22 de abril de 1938, una "ley de guerra" que en realidad duró 28 años. La prensa escrita viviria en un estado de permanente tutela hasta la Ley de Prensa e Imprenta — Ley Fraga— de 1966.

\section{LA PRENSA MADRILEÑA}

El Estado considera que debe contar con una prensa que defienda los intereses nacionales y cuyos fines no sean otros que los del propio Estado. Debe contribuir al fortalecimiento de éste y anteponer sus fines particulares a los del servicio al país. Este era el espíritu que latía en la Ley de 1938 y del que se desprendía un claro rechazo a la prensa pluralista.

Durante el régimen franquista coexistieron dos tipos fundamentales de prensa: la Prensa del Movimiento de fines más ideológicos que mercantiles, y la prensa de propiedad privada, en general periódicos que ya existían antes de la guerra y de ideología conservadora. Al desaparecer todos los periódicos de cariz liberal, radical, republicano, socialista $o$ anarquista tras la victoria nacional, se mantuvieron únicamente tres diarios privados: el monárquico $A B C$, Informaciones, con Víctor de la Serna al frente de la dirección y el católico Ya.

Arriba, creado como semanario en 1935 por José Antonio Primo de Rivera, reapareció como diario el 28 de marzo de 1939. Fue el ejemplo más significativo dentro de la llamada Prensa del Movimiento. Instrumento para la orientación ideológica del régimen y para el adoctrinamiento popular. En sus páginas se hacía eco de las premisas fundamentales: unidad de hombres y tierras de España, universalidad de destino, desprecio del comunismo ateo y de las democracias divisoras y defensa del nacional-sindicalismo. En este último aspecto Pueblo realizó un papel fundamental en los primeros años de la posguerra. Había nacido como Prensa del Movimiento en julio de 1940, limitándose prácticamente a ser portavoz oficial del sindicalismo del régimen. Pocos años después su propiedad pasó a la Delegación Nacional de Sindicatos. La relativa autonomía que esto podía suponer, unida a la dirección ejercida por Emilio Romero desde 1952 -salvo la breve interrupción de 1954 a 1956- cambió el carácter del periódico al que dio cierto aire de populismo y "aperturismo» crítico e informativo.

El panorama de la prensa madrileña se completa con otros dos periódicos significativos. Madrid, vespertino cuyo primer ejemplar se publicó el 
8 de abril de 1939, fue el único diario de propiedad particular y de nueva creación en la capital de España, permitido por el Gobierno. Esto se debió posiblemente a los méritos de Juan Pujol -experto periodista que asumió la dirección- al servicio de la causa franquista y que fue jefe del primer gabinete de prensa de la Junta de Defensa Nacional en 1936. Si la formación política de los ciudadanos correspondía fundamentalmente a la prensa del Movimiento, Madrid atendería sobre todo a la educación y afianzamiento de las costumbres siempre con un cariz tremendamente conservador que se mantuvo al menos hasta 1962 en que la familia Pujol decidió vender el periódico iniciándose para éste una nueva etapa.

El Alcázar surgió en 1936 durante el asedio de la fortaleza toledana. Pasó a editarse en Toledo y finalmente en Madrid al terminar la guerra. Su vida periodistica fue bastante lánguida hasta los primeros años sesenta cuando José Luis Cebrián asumió la dirección.

Si añadimos el resumen semanal ofrecido por La hoja del lunes y el diario deportivo Marca, se puede contemplar el panorama que dominó la prensa diaria madrileña desde el final de la guerra hasta 1966. A esto habría que añadir el conjunto de revistas, radio y televisión cuya programación regular se inauguró en octubre de 1956. En este breve estudio centraremos nuestra atención en la prensa escrita y diaria por contar con una sección fija de arte y profesionales permanentes responsables de la misma.

\section{EL CONTROL INFORMATIVO Y LA CREACIÓN DE UNA NUEVA CULTURA}

Como hemos dicho, el periodismo debía estar al servicio del Estado. Entre 1939 y 1966 la información estuvo totalmente controlada dando lugar a una prensa cada vez más uniforme y monótona. Los medios establecidos para esta rigurosa vigilancia tenían como punto de partida la mencionada Ley de Prensa de 1938 y se concretaban en la ley de censura previa y sus «hojas de inspección", las consignas, las medidas de control sobre los periodistas y la revisión de la información internacional a través de la Agencia EFE.

El terreno cultural estuvo quizás menos controlado que el político al considerarse menos comprometido posiblemente por no contar con una clara definición cultural del régimen.

Finalizada la guerra se hizo necesario elaborar una teoría clara que marcase las pautas por las que arte y cultura en general debían discurrir. Su uso como instrumentos de propaganda y difusión de los ideales sobre los que se quería fundar la nueva sociedad lleva a establecer una corres- 
pondencia entre arte y política. Esto se refleja en el discurso pronunciado por Rafael Sánchez Mazas nombrado ministro sin cartera en 1939, con motivo de la inauguración de la Exposición de Arte Mediterráneo, discurso en el que estableció la correspondencia entre arte, política y cultura ${ }^{2}$.

Con estas premisas, finalizada la guerra se inició la elaboración de una teoría sobre lo que el arte debía ser de cara a lograr la deseada nueva sociedad. Esta teoría se construyó sobre pilares falangistas con la colaboración de algunos críticos.

El corpus doctrinal tomó de Ortega y Gasset su pensamiento definido en La deshumanización del arte. De D'Ors tomaron la concepción de arte y cultura como un servicio a la sociedad. Su dimensión trascendente contribuiría a una mayor riqueza espiritual. Giménez Caballero y su explicación de la relación directa entre arte y política, fue probablemente el precedente más importante de las pautas que se iban a seguir ${ }^{3}$. Con estas directrices se establecieron las líneas por las que va a discurrir en el futuro el arte oficialmente admitido, y que luego, al iniciarse el cambio de actitud oficial en los años cincuenta, seguirán siendo los cauces de valoración de los sectores más conservadores de la crítica.

\section{CRITICA Y VANGUARDIA}

La década de los cuarenta fue una etapa de notable inseguridad a la vez que de un lento despertar artístico. Desde los sectores oficiales se señaló la decadencia del arte desde el impresionismo acentuado por el desarrollo de las vanguardias. El concepto de vanguardia no estaba definido con precisión. En conjunto era bastante general el desconocimiento de los acontecimientos artísticos exteriores. Al leer la prensa del momento se percibe la falta de eco de la evolución artística exterior así como una manera de valorar las obras de arte que se mantiene dentro de premisas académicas bastante anacrónicas. Se mantuvo una actitud de claro rechazo hacia unas vanguardias cosmopolitas y antinacionalistas que al romper con la figuración naturalista se apartaban en definitiva de la concepción realista de base aristotélico-tomista.

La recuperación de realismo y religiosidad serían las dos notas constitutivas del nuevo arte español y de la crítica correspondiente. Cecilio Bar-

Llorente hefnandez, Ángel, Arte e ideología en el tranquismo. Madrid, Visor, 1995, pág. 45. lbídem, pág. 34. 
berán, crítico de arte de $A B C$, reconocería la espiritualidad como algo consustancial al arte español ${ }^{4}$. Asimismo, Sánchez Camargo, catedrático de Literatura del Instituto San Isidro que ejerció la crítica de arte en el diario El Alcázar en esos años para pasar luego a ocuparse de esta sección en Pueblo y en La hoja del lunes, afirmaba que el arte debía expresar una espiritualidad al servicio de nobles metas ${ }^{5}$. A pesar de la actitud de oposición a la vanguardia desde el punto de vista ideológico, hubo algunas actitudes abiertas al reconocimiento de valores plásticos. Así, Benito Rodríguez-Filloy, crítico de arte de Arriba, escribió sobre la validez formal de las vanguardias y sus posibilidades expresivas. Sin embargo y en conjunto, el arte de vanguardia era considerado el resultado de la deshumanización el arte.

Aún así, y como dirá muchos años más tarde Castro Arines:

"Algo sucedía a la vez y era que la gente del arte en Madrid, que durante años vivió al pairo, dio en entender que "por fuera" del vivir encorsetado del arte nacional, eran próximas a la actualidad madrileña nuevas corrientes, nuevos pensamientos y nuevos hallazgos destinados a cambiar el oriente al arte de los cuarenta. Se empezaba a perder el miedo; se asentaban y afirmaban tímidas ideologías, y la gente del arte comenzaba como en los viejos tiempos de la Dictadura y de la República, a asociarse, agrupándose en tendencias más o menos afines a las vacilaciones propias del tiempo. No se debe olvidar que la década se distinguió en Madrid por sus modos de ocultación y provisionalidad" 6 .

Efectivamente, en este momento convergen diversos factores que contribuyen a esa «renovación». Por una parte la ruptura del aislamiento internacional a que había quedado sometida España que se traduce en el restablecimiento de las relaciones diplomáticas con Estados Unidos y la Santa Sede. En el interior se forma un nuevo Gobierno de carácter más aperturista. $Y$, desde el punto de vista artístico, el cambio que paulatinamente se había ido produciendo en la obra de algunos artistas llevó a la conquista de premios en los certámenes del extranjero precisamente a aquellos que apostaban por las nuevas tendencias de vanguardia ${ }^{7}$. Esto había despertado la atención de la crítica ya en los últimos años cuarenta. Camón Aznar empezó a advertir de los peligros de las «escuelas abstrac-

$A B C, 9-$ VII-1942.

El Alcázar, 16-XI-1939.

CASTRO ARINES, José de, "El arte en Madrid: los años críticos", en Madrid. Arte de los sesenta, Madrid, Comunidad de Madrid,1990, pág. 51.

Exposición de Arte Español Contemporáneo, Buenos Aires, 1947; Exposición de Arte Español, El Cairo, 1950; Bienal de Venecia, (Pabellón español), Venecia, 1950. 
tas" y del nuevo cariz "extoravagante" que se manifestaba en algunas creaciones ${ }^{8}$. El gobierno español seguirá haciendo un uso político del arte si bien es consciente de que debe adoptar una actitud diferente si desea lograr resultados efectivos. La actitud oficial parece apostar por la renovación plástica:

"Algo nuevo iba a tomar su impulso en el arte de Madrid: la aventura no figurativa, que sacudió en sus cimientos la arquitectura del arte nacional, a cuyo impulso las representaciones españolas en las Bienales internacionales comenzaron a significar el poder nuevo de nuestro arte por el mundo adelante (...). De cara al futuro, al arte "laudatorio», ya sin sangre, le había llegado su hora... ${ }^{9}$.

El primer acontecimiento en que este cambio se hizo oficialmente público fue en la Bienal Hispanoamericana de Arte celebrada en Madrid en 1951. El certamen dejaba completamente libre la entrada a las nuevas tendencias artísticas. Fue en este momento cuando los sectores más conservadores del arte español, desconectados de toda innovación, cómodamente instalados en su inalterable modo de hacer, dieron la voz de alarma temerosos de perder su dominio casi absoluto. Esto desencadenó una ardua polémica desarrollada en la prensa diaria que fue fiel reflejo de la escisión existente entre un sector de la crítica absolutamente académico y conservador representado especialmente por el diario Madrid y su crítico José Prados López, y aquellos que apostaron decidida y combativamente por la renovación artística. Camón Aznar, que habia sustituido a Cecilio Barberán en las páginas de $A B C$, Ramón D. Faraldo en su critica del diario YA, Figuerola-Ferretti en Arriba y Sánchez Camargo en Pueblo, se hicieron eco del acontecimiento en un tono en general positivo y de encomio siguiendo las directrices que en previa reunión les había dado Sánchez Bella, Director del Instituto de Cultura Hispánica ${ }^{10}$, y de la que informó el diario Pueblo ${ }^{11}$. En la reunión, además de distintos aspectos organizativos, otra cuestión muy discutida fue:

“...Si merecía darse a la Exposición una preponderancia concreta o abstracta. (...) En esta exposición se pretende demostrar que, junto a un

$A B C, 16 \cdot X I 1-1949$

Castro Arines, J. Op. cit., pág. 53.

${ }^{10}$ Sánchez Bella a pesar de esta actitud, años más tarde declaraba en el diario Madrid, 2-ł1954: “El arte abstracto es el que más se presta a falsedad. (...) Tiene un $95 \%$ de camelo (...). La Bienal de Sao Paulo constituye un auténtico fracaso". Esto prueba un desconocimiento real desde el punto de vista artístico y un apoyo que obedece a causas ajenas al propio convencimiento.

Pueblo, 9-V-1951. 
arte clásico, de ideales concretos ya consagrados, fermenta, se desarrolla y prospera en los estudios españoles ese arte denominado abstracto, que luego ha de asombrar al mundo con su audacia, su fantasía y su pasión lírica e intelectual»».

El hecho de que la crítica se reuniese bajo la presidencia de una autoridad oficial pone de manifiesto hasta que punto ésta carecía de independencia:

"Los redactores de los diarios prometieron colaborar estrechamente con los organizadores de la I Bienal Hispanoamericana de Arte».

En la reunión se señalaba el cariz de la exposición, de donde se desprendía la tendencia artística que debía apoyarse y qué debía sembrarse en la opinión pública. La crítica se comprometió incluso a la elección unánime del mejor pintor de la exposición al que premiarían con una serie de artículos sobre su obra.

De todo esto puede deducirse que tanto el arte como la crítica, dos realidades que es difícil separar en su evolución, entraron en una nueva etapa aunque siempre dentro de unos márgenes de ruptura bastante controlados. El campo de acción no se limita ya únicamente a una figuración -que en gran medida sigue conservando su dominio- y a artistas consagrados cultivadores de la misma. Hay una apuesta por la renovación que, si bien es limitada, permitirá una ampliación progresiva tanto en la creación plástica como en los comentarios de la prensa diaria.

Como ejemplos ilustrativos de la nueva situación podemos citar que los premios de la Bienal Hispanoamericana de Arte fueron otorgados a Benjamín Palencia por su obra «Paisaje»; a Vázquez Díaz se le concedió un premio a la totalidad de su Obra y a José Rebull el gran premio de escultura. En la exposición participaron tanto artistas de tendencias puramente académicas como otros de línea no figurativa mucho más radical. ¿Por qué es significativo este reparto de premios? Porque de algún modo marca la pauta oficial del momento y de cara al futuro. La elección de artistas cuya obra recoge claros signos de modernidad pero que no significan la ruptura total que hubieran supuesto propuestas no figurativas mucho más agresivas (Tapies, Millares, Planasdurá, Mampaso...). Así pues, a lo largo de la década, de un modo u otro, será constante en la prensa la referencia a los maestros o genios de la pintura española del pasado y del presente, de dentro y de fuera del país: Velázquez, Goya, el Greco o Zurbarán. Picasso, Dalí y Miró, fuera de España. Vázquez Díaz, Benjamín Palencia, Zabaleta o Angel Ferrant 
en el interior ${ }^{12}$. En general estos fueron los artistas más premiados, objeto de los más frecuentes homenajes y a los que se dedicaban con más frecuencia secciones especiales en los periódicos. Entre estas coordenadas se movió en los años cincuenta la crítica de la prensa cotidiana. Sus deseos de apertura a la modernidad seguían presididos por las palabras que pronunció Ruiz Giménez en la inauguración de la 1 Bienal Hispanoamericana de Arte:

"La forma superior de colaborar con los artistas es meter dentro de su espíritu los conceptos de Patria y de fe, inyectándoles la preocupación del servicio a la Patria y de la fe cristiana. (...) Los jóvenes artistas miran con fe y esperanza a su Caudillo, seguros de que la belleza presidirá todos los actos de la política, siendo una realidad la verdad, la belleza y el bien» ${ }^{13}$.

\section{LA FIGURA DEL CRÍTICO}

La crítica de arte la ejercian personajes procedentes de distintos campos de la cultura y de fidelidad ideológica garantizada. Camón Aznar era catedrático de Historia del Arte Medieval en Madrid desde 1948 y decano de la Facultad de Filosofía y Letras desde 1958, académico de la Real Academia de Bellas Artes de San Fernando y de la Real Academia de la Historia ${ }^{14}$ donde cubrió la vacante de Gregorio Marañón ${ }^{15}$. Dirigía la $R e-$ vista de ideas estéticas ${ }^{16}$ que publicaba el CSIC del que era consejero, y la revista Goya, publicación del museo Lázaro Galdiano del que también era director ${ }^{17}$. Esta revista combinaba la publicación de artículos de His-

\footnotetext{
12 Ejemplos en este sentido son los siguientes artículos:

Informaciones, 9-IX-1955

Hoja del Lunes, 10-V-1954: “Vazquez Diaz, maestro de guía espiritual”

Arriba, 13-VI-1953: Exposición homenaje a Vázquez Díaz en el Palacio de Bibliotecas y Museos.

Informaciones, 16-IV-1955: «Palencia se ha trocado en estos años últimos, de un pintor apoyado por la minoría, en un pintor al que la mayoría festeja y celebra (...) Palencia es hoy ya un clásico fiel a su ley..." (Castro Arines).

Ya, 31-111-1955: "Su veracidad es tan primordial que no la han desvirtuado ni la acción disolvente del arte moderno ni la acción de París. (...) Zabaleta ha aprendido todos los leguajes de la pintura moderna..." (Ramón D. Faraldo).

13 ABC, 13-X-1951.

14 Informaciones, $19-\times 1-1960$.

15 Madrid, 28-V-1963.

16 Pueblo, 2-XII-1952.

17 Arriba, 24-IX-1954: “Goya, una revista dirigida por Camón Aznar. (...) La garantía representada por la dirección de Camón Aznar, docta y mesurada, capaz de conseguir el equilibrio entre los aspectos más variados del arte..." (Figuerola-Ferretti).
} 
toria del Arte Antiguo con otros de mayor actualidad. Así, en un mismo número podía encontrarse un trabajo dedicado a los inicios de la pintura gótica junto a otro titulado "Angel Ferrant en la escuela contemporánea" de Luis Felipe Vivanco ${ }^{18}$. Era frecuente que representase oficialmente a España en las escasas pero crecientes ocasiones en que ésta era requerida ${ }^{19}$. Camón Aznar fue autor de numerosas obras a caballo entre la historia del arte y la filosofía. Fue quizás quién más claramente desarrolló entre los críticos del momento lo que podría llamarse «una filosofía del arte" ${ }^{20}$. El tiempo en el arte es un claro ejemplo de este tipo de actividad. Fue presidente de AICA ${ }^{21}$. En 1960 se creó AECA -Asociación Española de Críticos de Arte- cuya directiva coincidía con la de AICA a la que en esa fecha avanzada pertenecían ya figuras como Carlos Antonio Areán, Cirici Pellicer, Moreno Galván o Cirilo Popovici, que constituirian la base de la renovadora crítica de los sesenta ${ }^{22}$. En una entrevista publicada por el diario Arriba ${ }^{23}$ calificaba la pintura moderna como aun gigantesco avance en el mundo del arte" y la crítica de arte española "sencillamente maravillosa. Yo conozco la crítica mundial y, desde luego, no se puede comparar. No hay duda de que tenemos la mejor crítica del mundo. Se escriben páginas admirables, páginas que sin duda quedarán". Esta opinión era corroborada por el también crítico del diario Informaciones, José de Castro Arines ${ }^{24}$ que al referirse a la citada entrevista no solo expresó su acuerdo con Camón Aznar sino que añadió sus propios argumentos de apoyo: "La crítica de arte española es buena por múltiples razones y circunstancias. Primeramente porque tiene para ello la firmeza de su honestidad (...), porque no distingue a los artistas por las maneras de su hacer, sino por la bondad exclusiva de sus invenciones. Es buena en tercer lugar, porque se activa en la relación continua con el arte (...) y es que ella es buena en razón de que también el arte español actual es bueno. (...) Pero aún hay más, y es la labor educativa de la crítica, de en-

18 Informaciones, 4-X11-1954.

19 Informaciones, 19-VII-1954: aparece la noticia de que Camón Aznar representó a España en un acto oficial en París junto a García Sanchiz, donde pronunció una conferencia

20 "Camón Aznar filósofo del arte", Arriba, 8-XI-1958.

21 Arriba, 2-X1-1954: "Ha quedado constituida la sección española de la Asociación Internacional de Críticos de Arte, dependiente de la UNESCO. Está presidida por don José Camón Aznar (...). Figuran como miembros de la misma los señores Ainaud Lasarte, Gudioi Ricart, Lafuente Ferrari, José Gaya Nuño, Manuel Sánchez Camargo y Luis Figuerola-Ferretti. Como domicilio provisional el señor Camón Aznar ha ofrecido una dependencia del museo que dirige".

22 GAYA NUÑO,J.A. Historia de la crítica de arte en España, Bilbao, lberico Europea Ediciones. 1975

23 Arriba, 16-IX-1962

24 Castro Arines sustituyó a Cecilio Barberán en la sección de arte del diario Informaciones en febrero de 1953. 
señanza y orientación pública. Y la obra de investigación, de vigilancia, de exposición de los problemas vitales del arte actual...” ${ }^{25}$. En general la actitud de Castro Arines hacia las nuevas tendencias de la pintura era abierta y reflejaba cierta formación en esta línea. Así el diario Ya informaba el 27-V-1955 de la conferencia que pronunciaría Castro Arines en la galería Fernando Fe: "Notas sobre arte abstracto". Igualmente Madrid anunciaba el 22-VIII-1961 la publicación de su libro sobre Lucio Muñoz: «Castro Arines, crítico de arte de la hora actual, ha publicado un libro interesante acerca del pintor Lucio, en el que enjuicia de manera certera y honda de conocimientos y de propios criterios la expresión plástica de un pintor de hoy metido en abstracciones y en meditaciones...".

Otro personaje de la crítica cotidiana procedente de las filas de los historiadores fue Enrique Lafuente Ferrari, nacido en Madrid en 1898. Catedrático en la Escuela de Bellas Artes de San Fernando y miembro de Real Academia de Bellas Artes de San Fernando ${ }^{26}$, pertenecía también al cuerpo de Archivos, Bibliotecas y Museos y era director del Tesoro Artístico del Patrimonio Nacional. Hacía compatible por tanto su actividad profesional con otras múltiples actividades de carácter más o menos oficial y didáctico como la dirección del Museo de Arte Moderno ${ }^{27}$. En 1952 impartía un curso de pintura contemporánea, "Del modernismo a Dalí" ${ }^{28}$ cuyo título parece indicarnos los límites del conocimiento de la vanguardia que entonces se tenía en España. El 19 de enero de 1955 se inauguró en el Ateneo un "cursillo de diez lecciones de arte" donde Lafuente se extendió ya desde la crisis del arte del siglo XIX hasta el arte abstracto ${ }^{29}$. La crítica cotidiana la ejerció en el diario YA especialmente entre 1953 y 1955. En este periódico se combinaba la actividad de Lafuente Ferrari con la desarrollada por Antonio Cobos y Ramón D. Faraldo quedando los dos últimos a partir de 1956. Aún escribiendo en el mismo periódico y a veces en la misma página, los puntos de vista respecto a Cobos eran bastante diferentes. Así, en 1954 mientras Lafuente se lamentaba del apartamiento español "de la gran rueda de exposiciones internacionales" y afirmaba la existencia "de gentes deseosas de entrar en contacto con el arte, de estar al día y de alcanzar una actitud justa y comprensiva frente a los problemas de la expresión actual» ${ }^{30}$, Antonio Cobos decía: «En los años que precedieron a nuestra Cru-

Informaciones, 18-1X-1962.

Madrid, 20-11-1961, da la noticia de su nombramiento como académico de la misma.

$\mathrm{Ya}, 28-\mathrm{XI}-1958$.

Arriba, 16-111-1952.

Ya, 18-1-1955

Ya, I-1954 
zada, cuando en el mundo entero triunfaba el concepto pictórico de los Matisse, Braque, Picasso y De Chirico, en nuestra patria se cerraba a cal y canto el acceso a los certámenes nacionales de los jóvenes seguidores de esa pintura. Transcurrrieron los primeros años después de la Liberación, y (...) se olvidan de que en el mundo comienzan a pasarse los «ismos» y nos ofrecen un verdadero sarampión de lienzos surrealistas, abstractos y expresionistas» 31. Ramón D. Faraldo fue de los tres, el que prestó mayor atención a las corrientes más modernas de la pintura. Mientras en la misma página Antonio Cobos comentaba la exposición de Agustín Segura en la sala Cano ${ }^{32}$, Faraldo iniciaba su comentario sobre Genovés sometiendo a crítica a la crítica: "Por supuesto, la crítica de arte, o la literatura así llamada, se equivoca a menudo. Sobre todo cuando esforzándose en descubrir pintores que no sean el Greco, Goya o Picasso, dice de un joven más o menos desconocido que está bien, que es un pintor o será un gran pintor. Entonces se corre cierto riesgo. Hay quienes no lo corren nunca y se atienen a los consagrados. En cuanto a mí, prefiero incurrir en frecuentes errores y tener un acierto de vez en cuando, que vivir de las rentas de los genios seculares" ${ }^{33}$. En los años siguientes estas posturas se mantuvieron e incluso se radicalizaron y así mientras Faraldo escribía artículos como "Otro arte y un pintor verdadero" ${ }^{34} \mathrm{O}$ "En pleno furor abstracto ${ }^{35}$ y analizaba las exposiciones de las salas del Ateneo ${ }^{36}$ y de la Sala Negra del Museo de Arte Contemporáneo ${ }^{37}$, Cobos, iniciados los sesenta, seguía

\footnotetext{
Ya, 27-XI-1954

"Dominio absoluto en los lienzos de Agustín Segura", Ya, 10-III-1957, pág. 8.

"Genovés, un pintor casi maravilloso", Ya, 10-1II-1957, pág. 8.

Ya, 14-V-1957.
}

Respecto a esta exposición combiene aclarar su origen. En 1952 Michel Tapié organizó en París la exposición "Un art autre" que incluía la obra de más de cuarenta artistas incluyendo a estadounidenses como Pollock, Mark Tobey, Sam Francis además de Dubuffet, Fautrier y Wols. En la situación de posguerra Tapié consideró que "se necesitaban temperamentos dispuestos a romper con todo, cuyas obras eran inquietantes, pasmosas, llenas de magia y violencia para reencaminar al público" (Mozinska, Anna, El arte abstracto, Barcelona, Destino, 1996, pág. 128.) Las connotaciones de esta exposición se reflejaron en los años posteriores. Tapié presentó en Barcelona en 1957 la exposición "Otro arte" que luego en Madrid se exhibiría en la Sala Negra. Asi, Sánchez Camargo en Pueblo del 2-V-1957 se refería a la misma exposición que Faraldo - «Otro arte - de la que afirmaba que reunia las firmas de mayor renombre dentro del movimiento no figurativo: Burri, Falkenstein, Hossiason, Appel, Wols, Fautrier, Pollock, Wessel...entre los extranjeros, y Tharrats, Feito, Canogar o Tapies entre los españoles.

35 Ya, 18-XII-1958.

36 El Ateneo contaba con la sala Prado y en 1956 se inauguró otra sala para las nuevas tendencias.

Informaciones, 24-III-1956: “El Ateneo inauguró su nuevo salón de la calle Santa Catalina, un acierto de arquitectura ambiente...".

37 De su apertura daba noticia el diario Madrid, $7-\mathrm{V}-1957$, con una crítica que refleja la postura del periódico respecto a las nuevas tendencias: "Se ha abierto otra sala de exposiciones 
centrado en los Salones de Otoño o en la Antológica organizada por la Asociación Española de Críticos de Arte que «pone al desnudo el error que cometieron los que llevaron nuestro arte actual hacia el exterior por motivos más o menos «bienales» excluyendo de la representación española a los artistas encasillados en tendencias o estilos no gratos a los organizadores. (...) Cuando recordamos la horrenda monotonía conceptual, temática y técnica de las exposiciones colectivas de artistas europeos y americanos celebradas en los últimos años ${ }^{38}$, esta muestra ensancha gozosamente nuestro corazón» ${ }^{39}$.

En la misma línea de Antonio Cobos se desarrolla la labor crítica de Mariano Tomás en el diario Madrid. Había nacido en Hellín en 1890. Poeta, novelista, dramaturgo, biógrafo. Colaboró en La Esfera, Blanco y Negro y $A B C$. Fue cronista y crítico de arte único del diario Madrid hasta 1957 en que falleció siendo sustituido por José Prados López - Secretario Perpetuo de la Asociación de Pintores y Escultores ${ }^{40}$ y arduo organizador de los Salones de Otoño ${ }^{41}$ - que siguió totalmente la línea iniciada por su predecesor tal como expresó en el artículo con que inició su andadura crítica en Madrid: “Con la emoción que produce tomar en las manos la antorcha (...) que Mariano Tomás dejó al caer, comienzo yo mi labor,(...) prendido ya el corazón por las grandes verdades del espíritu que dicta Dios y no las mentiras de los hombres" ${ }^{42}$. En conjunto la postura tanto del diario como de sus críticos se resume en un claro rechazo de las tendencias abstractas cuyos acontecimientos son frecuentemente silenciados o si se citan es únicamente para criticarlos. En general no se da información sobre exposiciones y nuevas galerías de arte en las que se exponen estas

dependiente de la Dirección del Museo de Arte Contemporáneo - cuyos Director y Subdirector eran respectivamente Chueca Goitia y Sánchez Camargo-. Se titula Sala Negra y está situada en la calle de Recoletos número 2. La primera exposición que hemos visto está compuesta por telas y arpilleras sobre las cuales se ha pasado una brocha gorda. (...) No comprendemos que estas cosas se puedan colocar en un muro como adorno, cuando el mismo muro produce una sensación idéntica...”.

38 Desde 1955 se venian organizando desde la Dirección General de Bellas Artes y a través de la Dirección General de Relaciones Culturales del Ministerio de Asuntos Exteriores distintas exposiciones destinadas a dar a conocer la producción artística exterior. Así por ejemplo, en 1955 se concertó la celebración de una serie de exposiciones de ARTE UNIVERSAL CONTEMPORÁNEO que incluyó una exposición Retrospectiva de pintura estadounidense (Ya, 24-IV-1955), Tendencias recientes de la pintura francesa contemporánea (Madrid, 26-V-1955 y 1-V1-1955), Arte italiano contemporáneo (Madrid, 25-V-1955 y 10-Vl-1955), Pintura holandesa contemporánea (Madrid, 16 IV-1955) y dos muestras más de pintura alemana y de pintura nórdica.

39 Ya, 8-IV-1962.

40 Hoja del Lunes, 2-II-1953.

41 Pueblo, 2-XII-1954.

42 Madrid, 8-X-1957. 
tendencias ${ }^{43}$. También la misma crítica de arte es enjuiciada. Si Mariano Tomás había expresado su añoranza por una crítica de las características de la realizada por José Francés: "iQué lástima que a José Francés no le permitan sus muchas obligaciones deleitarnos con aquellos serenos y bien meditados resúmenes de cada ciclo de Exposiciones y que titulaba de ese modo! (...) Ahora quienes intenten acarrear materiales para estudiar la evolución, a veces revolución subversiva con gritos y alharacas, de nuestras artes plásticas, han de acudir a juicios más dispares y de menor fundamento" ${ }^{44}$. Prados López se refería al libro sobre Lucio Muñoz de Castro Arines en los siguientes términos: " Castro Arines en su libro trata de explicar lo que para nosotros no tiene explicación (...), hace esfuerzos sobrehumanos para lograr adeptos con una sutileza, una inteligencia y una belleza de forma que atrae y capta a conformes y disconformes (...) no por lo que dice sino por la manera como lo dice" ${ }^{45}$.

Manuel Sánchez Camargo era subdirector del Museo de Arte Contemporáneo ${ }^{46}$ y estaba vinculado a la Falange. Nació y murió en Madrid en 1911 y 1967 respectivamente. Antes que en Pueblo, ejerció las crítica de arte en Radio Nacional de España y en El Alcázar ${ }^{47}$. Logró gran prestigio en 1947 con su monografía sobre Solana. Figuerola-Ferretti comentó en Arriba del 24 de septiembre de 1954 su obra Pintura española contemporánea que editó el Instituto de Cultura Hispánica, «una reseña crítica de los protagonistas que componen la "Nueva Escuela de Madrid" (...) al márgen de tendencias determinadas, por un auténtico sentido de la sinceridad constructiva, diez pintores - F. Arias, Juan Guillermo, Enrique Herreros, F. Lorente, J. A. Morales, Picó, Redondela y E. Vicente- son mostrados...». Fue secretario de la Academia Breve de Crítica de Arte ${ }^{48}$. En 1963 publicó una historia de esta institución como homenaje a Eugenio D'Ors, - «al maestro Eugenio D'Ors a quien no pretendemos compararnos, como es lógico, y que, por otra parte, es el escritor más claro, ordenado y con trascendencia europea y buena, clásica raíz española que tenemos" ${ }^{49}$ - modelo probable de su modo de hacer crítica. Sánchez

43 Madrid, 11-VI-1953: "...la grotesca homogeneidad, el disparate repelente que se nos ofrece en la rotonda del Círculo de Bellas Artes. (...) Lo que hoy se exhibe en la Rotonda no merece otro comentario; ni siquiera escribir el nombre de la entidad que mancilla la gloria más pura de la pintura española al exponer tales engendros bajo su patronazgo".

44 Madrid, 30-VI-1953.

45 Madrid, 22-VIII-1962.

46 Madrid, II-1961.

47 Informaciones, 26-XII-1953.

48 Pueblo, 20-X-1954. El mismo afirmaba: "La buena fortuna en un tiempo nos correspondió con el cargo de secretario de la Academia Breve de Crítica de Arte...".

49 Informaciones, 26-XII-1953. 
Camargo afirmaba que la suya era una crítica sencilla, abierta a la generalidad del público: «En el quehacer diario, escueto, ingrato, informativo y bello del periodismo, solo pretendo que me entiendan el pintor, los aficionados y algunas personas de buena voluntad" en tanto que a las minorías selectas destinaba sus libros ${ }^{50}$. Su valoración del arte contemporáneo estaba dentro de las líneas generales que desde el principio hemos señalado. A Vázquez Díaz se refiere como "el maestro" en el año 1954. Fue uno de los críticos que siguiendo las pautas oficiales prestó su apoyo a las nuevas tendencias. En 1962, ya subdirector del nuevo Museo de Arte Contemporáneo hacía una valoración de la pintura española que puede resumir su actitud y casi también la evolución artística de la década:

«Si de algo podemos estar satisfechos, en relación con los valores espirituales, es el estado feliz de nuestra pintura, tanto en las resurrecciones espirituales del ayer (...) como en la primacía universal: Miró-Picasso-Dalí, y en las generaciones siguientes: desde Clavé a Tapies. Si a esto añadimos los premios internacionales de Sao Paulo, Pittsburg, Venecia: Oteyza, Ferrant, etc, el paisaje es para sentirse orgullosos como españoles... Además no faltan tampoco, para que el concierto sea perfecto, los ojos pueblerinos que miran el arte por una rendija... ¿Qué más? ${ }^{51}$ ”.

También Figuerola-Ferretti se puede clasificar entre los críticos de la corriente renovadora que a partir de los cincuenta propició el régimen, y más aún si tenemos en cuenta que desarrollaba su labor en Arriba, el periódico más representativo de la llamada prensa del Movimiento: "Creo haber demostrado en más de una ocasión mi simpatía por todo intento que pretende abrir nuevos oteros a la pintura...» ${ }^{52}$. Reconocía asimismo la necesidad de un nuevo enfoque de la labor crítica. El lenguaje tradicional así como el modo de valorar la obra de arte debían cambiar. Si entre el arte y su tiempo existe una estrecha vinculación, ésta se da también lógicamente en la relación con la crítica: «En una reproducción naturalista el crítico podia limitarse a glosar el resultado de una percepción en la fácil pintura puesta ante sus ojos (...). Ahora el crítico se enfrenta con una labor mucho más ardua porque prácticamente no existe "jurisprudencia", canon o receta práctica sobre la que cimentar su juicio si pretendemos valorar las nuevas manifestaciones del arte con independencia y justicia...” 53 .

\footnotetext{
Ibidem

Madrid, 2-II-1961

Arriba, 14-111-1953.

Arriba, X-1956.
} 
En 1953 Carlos Antonio Areán leía su tesis Doctoral sobre Basterra ${ }^{54}$. En esos momentos este dato no pasaba de ser una breve nota en la prensa cotidiana. Pero años más tarde reaparecía este nombre con otras publicaciones ${ }^{55}$ y como autor de distintos artículos. En realidad en los últimos años cincuenta, junto a los críticos habituales y a las secciones fijas, tiene lugar en la prensa diaria la intervención esporádica de figuras como Aguilera Cerni ${ }^{56}$, Moreno Galván ${ }^{57}$ o Cirici Pellicer ${ }^{58}$. Se puede hablar de ellos como una "nueva crítica» que se va a manifestar sobre todo en revistas especializadas: Acento, Artes, Estafeta Literaria, Aulas 63... Constituyen una nueva generación que no proceden de las filas de la Falange ni del genuino franquismo de posguerra. Su formación artística es mucho más sólida y está basada en un profundo conocimiento de las vanguardias internacionales y en el acontecer artístico exterior de su tiempo. En este sentido es significativo citar la serie de artículos de fondo sobre arte contemporáneo de Cirilo Popovici publicados en Arriba especialmente a lo largo de $1961^{59}$. En su mayoría apostarán por las nuevas tendencias y por la evolución natural del arte sin seguir premisas orientadoras oficiales. Algunos incluso serán clandestinos colaboradores antifranquistas como Moreno Galván o Aguilera Cerni ${ }^{60}$. Se abre una nueva etapa con un panorama de la crítica mucho más complejo.

54 Arriba, 29-XI-1953.

55 Pueblo, 28-XI-1961: "Veinte años de pintura de vanguardia en España, C. A. Areán ha escrito un libro necesario y preciso...". En Pueblo del 12-VI-1962 y 6-VII-1962 se citan otros trabajos suyos de crítica de arte.

56 Ya, 4-Xl-1964.

57 Arriba, 19-11-1961: informa sobre la publicación de la obra de José María MORENO GALVÁN, Introducción a la pintura española actual.

$58 \mathrm{Ya}, 13-\mathrm{XII-1961.}$

59 Popovici, C. "Arte, pueblo y masa", Arriba, 7-V-1961. "Hablar de arte", Arriba, 28-V-1961. "Arte y crisis", Arriba,4-VI-1961. "Arte y crisis: concepto de generación", Arriba, 11-VI-1961. "Paradoja de lo bello", Arriba, 18-VI-1961. "Arte y crisis", Arriba, 25-VI-1961 y 2-VII-1961.

"Actualidad de un arte pretérito", Arriba, 30-VII-1961. "Diálogo de sordos", Arriba, 24-IX-1961. "Actualización de Goya", Arriba, 29-X-1961. "En torno a dos bienales", Arriba, 12-X|-1961.

"Falsa actualidad", Arriba, 19-XI-1961. "Arte, imagen y materia", Arriba, 26-XI y 3-XIi-1961.

"En torno al arte aformal», Arriba, 17-XII-1961. "Berruguete, el español», Arriba, 17-XII-1961.

60 BOzAL, Valeriano: Arte del siglo xx en España. Pintura y escultura 1939. 1990. Madrid, Espasa Calpe, 1995, pág. 368. 


\section{PUBLICACIONES PERIÓDICAS CONSULTADAS COMO FUENTES} (1950-1963)

$A B C$. Diario ilustrado. Madrid.

Arriba. Órgano de Falange Española Tradicionalista y de las JONS de Madrid. Hoja del Lunes. Semanario. Madrid.

Informaciones.Diario. Madrid.

Madrid. Diario. Madrid.

Pueblo. Diario del Trabajo Nacional. Madrid.

Ya. Diario. Madrid.

\section{BIBLIOGRAFÍA}

AA.VV. España, vanguardia artística y realidad social, 1939-1976. BarceIona, Gustavo Gili, 1976.

- Madrid, arte de los sesenta. Madrid, Comunidad de Madrid, 1990.

- Del surrealismo al informalismo. Arte de los años cincuenta en Madrid. Madrid, Comunidad de Madrid, 1990.

Barrera, Carlos, Periodismo y franquismo. De la censura a la apertura. Barcelona, Ediciones Internacionales Universitarias, 1995.

Bozal, Valeriano, Arte del siglo xx en España. Pintura y escultura 19391990. Madrid, Espasa Calpe, 1995.

Gaya Muño, J. A. Historia de la crítica de arte en España. Bilbao, Ibérico Europea ediciones, 1975.

Llorente Hernández, A. Arte e ideología en el franquismo (1936-1951). Madrid, Visor, 1995.

MoszynSkA, A. El arte abstracto. Barcelona, Destino, 1996.

Sinova, J. La censura de prensa durante el franquismo. Madrid, Espasa Calpe, 1989. 
